\title{
Control of sexually transmitted diseases: View from the United States of America
}

\author{
WILLARD CATES JR, WILLIAM C PARRA, AND STUART T BROWN \\ From the Division of Sexually Transmitted Diseases, Centers for Disease Control, Atlanta, Georgia, USA
}

SUMMARY Past sexually transmitted disease (STD) control efforts in the United States of America have generally permitted a timely response to changes in intervention technology, antibiotic resistance, public funding, and media interest. ${ }^{2}$ Today, however, the expansion of STD organisms and syndromes at logarithmic rates has taxed our traditional labour intensive control approaches. We describe briefly the history of STD control strategies in the United States, discuss the seven components upon which current efforts are based, and speculate about our future programme initiatives.

\section{History}

National programmes to control STD were established in the USA during the early days of the first world war. ${ }^{1}$ For the next half century the focus was almost exclusively on the control of syphilis and its complications. Federal grants to support STD control initiatives were begun in 1939. Rapid treatment centres for syphilis and gonorrhoea were the second world war responses to a perceived STD problem. Widespread availability of penicillin led to a dismantling of the rapid treatment centres and a dissolution of the clinical specialty of venereology, which had largely been syphilology. During the 1950 s and 1960s, however, federal assistance continued to support the tracing of sexual partners, serological screening, and patient education services. The STD "epidemiologist" emerged as a central figure in syphilis control efforts.

By the late 1960s officials had become concerned with the rapidly escalating number of cases of gonorrhoea. Selective culture media stimulated the development of a national gonorrhoea control strategy. ${ }^{2}$ Pilot projects were undertaken in six American cities to identify infected women and also to treat the sexual partners of infected men. These projects showed the feasibility of such an approach. In 1972 the federal government dramatically increased its financial assistance to STD control. At the expense of syphilis, gonorrhoea gradually received a larger portion of the federal STD grant during the re-

Address for reprints: Dr W Cates, Director, Division of Sexually Transmitted Diseases, Center for Prevention Services, Centers for Disease Control, Atlanta, GA 30333, USA

Accepted for publication 21 February 1984 mainder of the decade. By 1982 estimated expenditures for gonorrhoea control accounted for almost three quarters of the federal STD grant.

Table I shows the national efforts for the control of gonorrhoea that evolved through three overlapping phases during the 1970 s. $^{3}$ Initial programme objectives sought to lower the incidence of disease and arrest the resistance of Neisseria gonorrhoeae to antibiotics. Asymptomatic women were the targets, and ineffective treatments were discouraged. By 1975 the control programme had entered a middle phase, emphasising more focused screening of high risk patients and more intensive follow up of treatment failures. The emergence of $\beta$-lactamase producing $N$ gonorrhoeae (PPNG), however, warranted the greatest programme concern. Active intervention with those patients likely to be transmitters was intended to improve the referral of sexual partners. Identification of priority targets was especially important as the larger numbers of patients with gonorrhoea precluded the intensive follow up of each patient, which had been possible in the syphilis era. ${ }^{2}$ By 1979 the strategies for the control of gonorrhoea had entered a third phase. Analyses of national data, as well as programme experiences, identified the importance of pelvic inflammatory disease (PID) in public health. Thus programme objectives expanded to reduce the incidence and limit the consequences of PID. ${ }^{4}$ Asymptomatic men who were partners of women with PID were actively sought. Hospital emergency rooms became additional places in which to seek people with gonorrhoea.

In the late 1970s the importance of STDs other than syphilis and gonorrhoea became recognised not only for their aetiological role in PID, but also for 
TABLE I Components of gonorrhea control in the United States from 1972 to $1983^{3}$

\begin{tabular}{lll}
\hline Phase I (1972-1975) & Phase II (1975-1979) & Phase III (1979-1983) \\
\hline (1) Identify asymptomatic women & (1) Identify high risk patients & (1) Recognise and manage PID \\
(2) Improve the quality of cultures & (2) Control resistant organisms & (2) Identify asymptomatic men \\
(3) Use effective treatment & (3) Emphasise test of cure & (3) Use hospital emergency rooms \\
\hline
\end{tabular}

their contribution to a variety of other syndromes causing patients to seek care at STD clinics. ${ }^{5}$ Control strategies increasingly sought to upgrade clinical skills, clinic services, and physical facilities to encourage more effective patient recruitment and additional ability to diagnose the broad array of STDs. Thus during the 1970 s we saw concern evolve from the firm base of "venereal" diseases to consideration of the vast range of the "STDs."

\section{Current STD control strategies}

To encourage international action in the control of STD the World Health Organisation. (WHO) identified seven components of STD intervention strategies, ${ }^{6}$ each of which is being used in the USA. They are as follows: (1) health education and promotion; (2) detection of disease; (3) appropriate treatment; (4) partner tracing and patient counselling; (5) evaluation of clinical services; (6) professional training; and (7) research.

\section{HEALTH EDUCATION}

Health education has been generally relegated to a second-class citizenship by STD professionals. This may have been because traditionally "scare tactics" based on judgmental messages have been ineffective in changing sexual behaviour. In fact, stigmatising infected patients by widespread social disapproval may even have hindered control of disease by delaying treatment. Recent STD education approaches, however, have promoted individual decision making in a more non-judgmental manner. These community health education efforts have encouraged primary preventive behaviour (before symptoms appear) in healthy persons at risk. For example, in today's more permissive society, messages which emphasise discriminative sexual intercourse, rather than proscribing coital activity altogether, may have already had an impact. In response to increasing concern with genital herpes in the USA, over half the unmarried people who believed themselves to be at risk reported changing their sexual behaviour to avoid this disease. ${ }^{7}$ Similar practices may have occurred in the gay community concordant with the recommendations of the Centers for Disease Control (CDC) for prevention of the acquired immune deficiency syndrome (AIDS). ${ }^{8}$
To date STD education has mainly contributed to secondary prevention, namely through advice directed to infected people after symptoms appear. Messages to these people encourage the following behaviour: prompt attendance for examination after exposure to high risk situations; early medical examination when symptomatic; compliance with oral treatment; and facilitation of other intervention such as referral of sexual partners. Other education strategies to increase the knowledge and awareness of STD in the general public have also been stressed in recent years. Prototype school curriculum materials for grades 6-12, based on a self instructional format, have been field tested; these will facilitate systematic STD education as part of family life and sex education courses throughout the country. A federally sponsored STD national hotline has been implemented since 1980 to provide both general and referral information to people interested in learning about STD. ${ }^{9}$ Thus health education can have a wide role in STD control strategies.

DETECTION OF DISEASE

Early detection of disease is an essential component of STD control strategies and takes place at two levels; at the STD clinic and in the community. At the STD clinic, identification of infected people by sensitive diagnostic techniques, combined with rapid treatment, reduces complications of disease and minimises further transmission. In the community, specific screening forms the basis for detection of disease in asymptomatic people and also identifies the groups at highest risk on which to focus further programme efforts. Whether for making specific diagnoses in those with symptoms, or for screening persons without symptoms, diagnostic tests for STD should ideally be rapid, inexpensive, simple, and accurate.

Accurate diagnosis is the sine qua non for the early detection strategies of STD control. The usual variables for assessing diagnostic accuracy-sensitivity and specificity-have a slightly different importance in STD control than in the screening of chronic conditions, ${ }^{10}$ largely because STD treatment is generally shorter and safer than that for other conditions. Sensitivity (reducing false negatives) consequently takes on an increased importance because missed cases place the infected person at continued risk of 
more serious complications, and result in further disease dissemination. Specificity (reducing false positives) is less important because treatment of a small percentage of unaffected people is associated with low morbidity, cost, and inconvenience. With STD, however, the human and emotional costs of erroneously stigmatising any person means that specificity cannot be neglected. Moreover, the public health costs of interviewing and tracing large numbers of false positive patients would drain limited STD resources.

Advances in laboratory techniques heralded previous major initiatives in STD control. For example, serological tests improved the diagnosis of syphilis, and selective culture media allowed screening for gonorrhoea. The decision to adopt or abandon a new diagnostic test is generally dictated by the prevalence of the disease in the population being screened and by the accuracy of the test. For instance, the prevalence of syphilis has become so low in the USA that the cost effectiveness of serological screening in some situations (such as in premarital examinations and new admissions to hospitals) is being debated. ${ }^{11} 12$

Yield and cost considerations are particularly important to screening activities, and selection of the target population greatly influences these factors. In the USA gonorrhoea programme, the cost per case diagnosed varied from $\$ 25.00$ in metropolitan STD clinics to as much as $\$ 350.00$ in areas of very low prevalence. ${ }^{13}$ When more selective screening was instituted in one location in 1976, the proportion of positive tests went from $4.5 \%$ to $8 \cdot 1 \%$ in one year, and the cost per case diagnosed was considerably reduced. ${ }^{14}$

Targeted screening has been implemented in some high risk groups such as homosexuals, prostitutes, and pregnant women. For example, in some countries Gram stained smears for gonorrhoea are used in compulsory screening of legalised or controlled prostitutes. The yields are usually low, however, and the impact is limited by the insensitivity of the Gram stain. For stemming specific outbreaks, however, screening of prostitutes for PPNG has proved useful in the USA. ${ }^{15}$

\section{APPROPRIATE TREATMENT}

Once the diagnosis is made, treatment should be inexpensive, simple, safe, and effective. Early and adequate treatment of patients and their sexual partners is an effective means of preventing both the resistance of STD organisms to antimicrobials, and the spread of STD. ${ }^{10}$ To improve the likelihood of physicians prescribing effective treatment, two approaches have been used in the United States: laboratory monitoring of STD isolates for antimicro- bial susceptibilities, ${ }^{16}$ and consensus groups to develop standard treatment recommendations. ${ }^{17}$

Studies correlating resistance to antibiotics with increasing treatment failure rates for a given antibiotic dose have been reported. ${ }^{16}$ Widespread use of suboptimal doses of antibiotics for treating gonococcal infection has led to selection of resistant chromosomal mutants in developing countries throughout the world. ${ }^{18} 19$ Thus penicillin has to be used either in higher doses ${ }^{20}$ or with a second drug. ${ }^{18}$ The recent outbreak in the USA caused by a nonPPNG strain of $N$ gonorrhoeae that is highly resistant to penicillin ${ }^{21}$ raises the possibility that such organisms may be widely distributed, but unrecognised, even in developed countries.

The emergence of a plasmid mediated $\beta$-lactamase producing strain of $N$ gonorrhoeae posed an even greater problem for STD control strategies in the USA. ${ }^{22}$ Although disc testing allows relatively rapid laboratory identification of these strains, the sharp increase in the reported number of PPNG cases between 1980 and 1982, as well as the greater expense of treating and tracing people infected with these strains, taxed the limited resources of local STD control programmes.

To increase the likelihood of successful treatment regimens for specific diagnoses the United States Public Health Service has established treatment recommendations as a standard part of its control strategy. These recommendations initially covered syphilis and gonorrhoea but have recently been expanded to include 18 other STDs and syndromes. ${ }^{1723}$ In the USA the process of achieving consensus among a group of clinicians expert in STD proved useful in establishing treatment guidelines that are applicable to diverse communities and flexible to accommodate alternative treatment regimens.

Selective prophylactic (epidemiological) treatment also has a major role in USA strategies for STD control. In certain instances it is inappropriate to wait for confirmation of specific diagnosis before starting treatment. Antibiotics should be administered when the diagnosis is considered likely, based on epidemiological indications, before infection is confirmed by laboratory methods. Thus selected groups of patients at high risk of infection are identified by epidemiological analyses and treated before confirmation of their infection. This affects the control of STD in three ways: it interrupts the chain of transmission between the time of testing and treatment; it ensures treatment for infected women with false negative endocervical cultures; and it guarantees treatment for those who might not return when notified of positive tests. Although this approach results in treating a percentage of uninfected people, it increases the chances of 
disrupting transmission, which is a crucial aspect of STD control. This approach of selective prophylactic treatment has recently effectively limited outbreaks of PPNG and chancroid in metropolitan areas of the USA. ${ }^{15} 24$ An adaptation of the same philosophy was recently used to recommend giving tetracycline concurrently with penicillin to patients with confirmed gonococcal infection, ${ }^{17}$ as a relatively high proportion are liable to be harbouring coexistent chlamydia organisms.

\section{PARTNER TRACING AND PATIENT \\ COUNSELLING}

Three general ways exist to trace sexual contacts (table II). STD control programmes in the United States have traditionally assumed that patients play relatively passive roles in disease control and prevention. Efforts to identify and ensure the treatment of sexual partners of infected patients therefore emphasised active intervention by the health providers by interviewing the patient, locating all named contacts, and assuring that these contacts were evaluated and treated. ${ }^{12}$ During the 1970s, however, this process of active intervention was modified in some settings to include a more simplified approach. Instead of relying solely on the health worker, the patient was directly encouraged to assume responsibility for locating and referring his or her sexual partners. 25 This self referral method, actively involves patients in the disease control effort, is inexpensive, is normally acceptable to patients, and reserves scarce staff time for other activities. Its potential shortcomings, however, include limited effectiveness, difficulty in evaluating its outcome, and non-productivity with unreliable patients. Under most circumstances some form of simplified tracing will be cost effective because it will lead with relatively little effort to a sizable number of infected people being treated.
Formal tracing using staff is more expensive and is therefore reserved for occasions when the cost is warranted or there is no cheaper way of achieving the same outcome. Occasions when this costly strategy has been useful include: (1) the introduction of a serious disease like infection with PPNG strains into a community previously unaffected ${ }^{26}$; (2) patients with gonococcal PID, in which a large proportion of male partners are free of symptoms ${ }^{27}$; (3) patients with repeated STD infections ${ }^{27}$; (4) female consorts of patients with infectious syphilis; and (5) children with STD infections.

During the past several years another partner referral approach has been used which is intermediate between self referral and professional contact tracing, which is called conditional contracting. ${ }^{28}$ This approach has several unique features and implies selection of the most reliable patients by health workers. A patient who is not interested, lacks communication skills, or is hostile towards his or her sexual partners is not a suitable candidate for conditional contracting. Appropriately motivated patients can choose to refer some or all of their sexual partners. A key task of the health worker is to help patients decide how to present information to partners acceptably and thus obtain the desired result. The staff not only sets conditions for the referral, helping patients understand why they should participate in the process, but also reminds them that partners must be examined promptly (usually within 24-48 hours). Patients must be aware that action by health workers remains a viable option for ineffective or uncooperative referrals.

A further shift in this strategy of disease control focuses on the counselling (or educating) of patients to facilitate changes in their behaviour. ${ }^{28}$ The traditional focus of partner tracing-bringing sexual partners to treatment - has been expanded to encourage: taking medication as directed; returning for

TABLE II Referral options for tracing sexual partners

\begin{tabular}{|c|c|c|}
\hline Option & Advantages & Disadvantages \\
\hline $\begin{array}{l}\text { Self Referral } \\
\text { Total patient responsibility; no } \\
\text { identification of consorts requested; } \\
\text { appointment cards provided to patient } \\
\text { for distribution to sexual partners. }\end{array}$ & Reduces cost, requires less staff time. & Difficult to evaluate and monitor. \\
\hline $\begin{array}{l}\text { Health provider referral } \\
\text { Health provider assumes total } \\
\text { responsibility for sexual partner referral. }\end{array}$ & $\begin{array}{l}\text { Maintains patient anonymity, monitoring } \\
\text { and control by health department. }\end{array}$ & $\begin{array}{l}\text { Increases cost; patients prevented from } \\
\text { helping in referral process. }\end{array}$ \\
\hline $\begin{array}{l}\text { Conditional coniracting } \\
\text { Health professional offers patient the } \\
\text { option to have partners referred by } \\
\text { health personnel or by themselves } \\
\text { within specified time; option is offered } \\
\text { only if the patient appears to be } \\
\text { interested, responsible, and able; } \\
\text { identification of consorts required. }\end{array}$ & $\begin{array}{l}\text { Monitoring and evaluative systems } \\
\text { assure that epidemiological treatment is } \\
\text { used; reduces cost; encourages patient } \\
\text { participation. }\end{array}$ & $\begin{array}{l}\text { Lacks anonymity; patient may not comply, } \\
\text { thus some time lost in the referral process; } \\
\text { costs are intermediate. }\end{array}$ \\
\hline
\end{tabular}


follow up tests; assuring examination of sexual partners; reducing transmission by avoiding exposure until the follow up is completed; and preventing exposure by using protection in high risk settings; and, if all else fails, responding to suspected disease by seeking appropriate medical evaluation promptly.

\section{EVALUATION OF CLINICAL SERVICES}

Adequate clinical management of patients and their sexual partners is the final pathway to control of STD. In the USA, however, clinical services vary widely and are often inadequate to provide appropriate care for patients with STD. For many years the public STD facilities were relegated to a lower class status. ${ }^{2}$ To enhance their image, our recent control efforts have sought to upgrade the physical appearance and capacity of the STD clinics and to improve the consideration of patients within these clinics. For example, a patient flow analysis may lead to more efficient use of existing resources. ${ }^{29}$ Redistribution of room use may provide greater privacy for patients during interviews and examinations; working hours can be changed to improve services for working patients during evening or early morning hours; assignment of specific tasks to existing workers may increase their efficiency and decrease staff friction.

The content of clinical care is an important determinant of outcome. To assist clinicians in making diagnoses and providing adequate treatment, use of simplified patient management protocols have been widely encouraged in the USA. ${ }^{30}$ These practical summaries are designed as wall charts and describe typical clinical presentations, criteria for diagnoses, appropriate treatments, and practical prevention points. They are useful in both public and private health care settings. The quality assurance guidelines have expanded on these STD summary medical protocols to cover the subjects of structure and management of clinics and patient education. ${ }^{31}$

Continued improvement of STD clinical services has also been stimulated in the USA by systematic programme reviews, which are largely based on the quality assurance guidelines. Personnel from all levels of government generally participate in these comprehensive clinic evaluations. They may focus on the structure (by listing staff and their qualifications and other resources), the process of care (by record audit and direct observation), or the outcome of the service (generally by record audit). The specific outcome of importance may vary from different points of view. For the patient the major concern is alleviation of symptoms or resolution of his or her problem. For the clinician an acceptable outcome may be to diagnose and treat a patient correctly. For the clinic administrator the outcome may be an encounter that is efficient in terms of time and cost.
From the STD control viewpoint, however, the outcome will include all the above concerns and will also consider the public health aspects of disease transmission and the prevention of complications in both the individual and the community.

\section{TRAINING}

Medical school curricula in the USA have not kept pace with the increasing incidence of STD over the past decade. ${ }^{32}$ This has created a dearth of clinicians with the necessary skills to diagnose, treat, and prevent STD. Both the public and private sectors have suffered from this neglect. Thus an integral part of STD control strategies in the USA has been to develop a coordinated system for training health care providers in the rapidly changing field of STD. This training affects workers in all specialties concerned with STD control-clinicians, laboratory workers, managers, field investigators, and health educators. For each group, both formal education systems and continuing in service training opportunities are essential.

The training initiatives have taken several approaches. To respond to acute needs, the quality assurance guidelines ${ }^{31}$ were developed to assist STD facilities to improve patient management. The STD treatment recommendations are regularly updated to reflect changing diagnostic capabilities, susceptibilities to antibiotics, and pharmacological innovations. ${ }^{17}$ Both these documents provide public health professionals with access to standardised clinical guidelines which can be adapted to their local needs. Another major professional training initiative has created regional STD prevention and treatment centres. ${ }^{33}$ Each centre is based on the integration of a university medical school with a model public STD clinic to offer training to mid-career health care providers, as well as medical, nursing, and paramedical students. Ten such multidisciplinary centres were in operation by 1983 and over 6500 students have been cumulatively trained since 1979 . Further development of these centres will allow them to servie as a focus for testing new methods of disease detection, evaluating recent treatment recommendations, and expanding patient management protocols.

\section{RESEARCH}

Both basic and applied research are crucial to STD control. Major changes in intervention strategy have accompanied the introduction of new antibiotics (such as penicillin), the development of better detection methods (such as Thayer-Martin media), the identification of resistant organisms (such as PPNG), and the recognition of high risk groups (such as homosexuals, patients with repeat infections, and 
women with PID). At present, quantum leaps in STD control programmes await the availability of rapid and inexpensive diagnostic tests for chlamydia, safe and effective treatment of genital herpes, and effective vaccines against herpes and gonorrhoea. Most STD research in the United States is supported by the National Institute of Health (NIH) and the Centers for Disease Control (CDC), ${ }^{34}$ although an increasing amount is funded either by voluntary organisations concerned with the magnitude of the STD problem or by private firms aware of the potential market. The objectives of this research are to characterise the pathophysiology, host response, and epidemiology of STDs to allow the further development of improved diagnosis, treatment, prevention, and control strategies.

The NIH supports a variety of basic scientific and clinical projects through the National Institute of Allergy and Infectious Disease and the National Institute of Child Health and Human Development. These include the development of gonococcal and herpes vaccines, investment in experimental clinical trials with antiviral agents, the identification of herpes transmission patterns within communities, and the determination of host responses to chlamydial infections. The NIH also sponsors training of postgraduate fellows in clinical and microbiological STD research and has undertaken periodical workshops to update the most rapidly advancing areas of STD knowledge. The private sphere has supported both basic and applied research in diagnosis (non-culture methods for detecting gonorrhoea), treatment (new antibiotics for resistant organisms), and prevention (vaccines).

The CDC, through the Division of Sexually Transmitted Diseases and the Sexually Transmitted Disease Laboratory Programme, conducts more applied research than the NIH. This is directed towards monitoring practical results and evaluating control strategies, and involves: clinical research, including field evaluation of recommended treatments for their efficacy and safety and of diagnostic tests for their sensitivity and specificity; epidemiological studies of the aetiology, natural history, clinical characteristics, demographic correlates, and geographic distribution of STDs; programmatic investigations to develop new methods of STD control; and related social science studies of STD behaviour patterns and motivations.

\section{The future}

After several decades of apathy and neglect, STDs are finally becoming recognised for the magnitude of illnesses they cause, the intensity of human suffering they generate, and the amount of health resources they consume. To emphasise how STDs have emerged as a primary health concern in the USA, the Surgeon General designated them as one of fifteen health priority areas for national prevention and control efforts. ${ }^{35}$ To achieve the broad national goal of reducing overall death rates and days of disability, specific national prevention objectives have been systematically established for STD. The targets for 1990 prevention objectives include reductions in the prevalence of gonorrhoea, gonococcal PID, primary and secondary syphilis, and congenital syphilis (table III). These targets are measurable and can be tracked relatively easily. Other 1990 objectives were based on estimations and include reductions in the prevalence of neonatal herpes, chlamydial pneumonia, and non-gonococcal urethritis and an increase in the percentages of couples using condoms or barrier methods for primary protection, the percentage of high school students receiving accurate STD education, and the percentage of clinicians able to diagnose and treat STDs. Although some progress has been made in achieving these objectives, far more must be done if the 1990 goals are to be reached.

Future strategies for STD control will have to build on the successful foundation of the past and to evaluate innovative approaches which are cost effective. To do this in the face of the greater range of STDs and the limited pool of resources, health consumers (especially patients with STD) will have to play a greater part in the intervention process. Moreover, both primary and secondary prevention must be emphasised.

Refinements of the WHO's seven time honoured control measures may make them even more effective. For example, STD education in high schools could stress the development of simplified reproductive life plans, which would dramatise the role of individual choice to decrease STD risks. ${ }^{36}$ Such health promotion mesages would be a routine part of family life and sex education courses, and they could emphasise appropriate use of contraceptives to

TABLE III Objectives for sexually transmitted diseases in the USA by $1990^{35}$

\begin{tabular}{lcc}
\hline & \multicolumn{2}{c}{ Prevalence in: } \\
\cline { 2 - 3 } & 1983 & 1990 \\
\hline Objectives & & \\
Gonorrhoea (/100 000 people) & 405 & 280 \\
Gonococcal PID (/100 000 people) & 117 & 60 \\
Primary \& secondary syphilis (/100 000 people) & 14 & 7 \\
Congenital syphilis (/100 000 live births) & $3 \cdot 1$ & $1 \cdot 5$ \\
Other Objectives & & $8 \cdot 5$ \\
Neonatal herpes (/100 000 live births) & 360 \\
Chlamydial pneumonia (/100 000 live births) & & 770 \\
Non-gonococcal urethritis (/100 000 people) & & $25 \%$ \\
Condom use & & $100 \%$ \\
STD education in high school & & $95 \%$ \\
STD knowledge in clinicians & & \\
\hline
\end{tabular}


prevent both STD and unintended pregnancy. For example, the condom or spermicidal sponge should be used in specific high risk settings, and oral contraceptives can prevent PID. Such messages can now even be aired in contraceptive commercials because the code banning the advertising of non-prescription contraceptives has been lifted. Broadcasting this information would be especially useful for getting messages to teenagers, as they spend a great deal of time watching television or listening to the radio.

The availability of non-culture diagnostic techniques for chlamydia will undoubtedly facilitate a national control programme. ${ }^{37}$ Effective oral antiviral agents for herpes simplex virus will promote control efforts against this organism. Identification of the variables affecting the cost effectiveness of different interview and investigation approaches in the management of gonorrhoea will permit conservation of future resources.

We must stay abreast of technological advances by expanding STD information systems to bring the control programmes into the computer age. ${ }^{38}$ To carry out STD control responsibilities successfully, programmes must collect, collate, and utilise data from patient medical records, case investigative documents, records of laboratory analyses, and case reports. Most current record systems in the USA are manually maintained; such systems are cumbersome, often inefficient, and difficult to link. During the remainder of the decade, state and local health departments should aim to install computer systems that improve the management and operational capabilities of STD control programmes. Obviously plans for the hardware and software components of any data system require that STD programme personnel are "computer literate."

Another priority is the development and evaluation of pilot STD curricula in medical schools. Although the STD prevention and training centres have been a very important first step in training qualified practitioners, we still need to reach clinicians during their initial formal training. In this way, we could create a cadre of STD specialistsmedically qualified clinicians who have a knowledge of, and a career commitment to, the STD specialty. If we developed such specialists, they would be capable of establishing their own training programmes and thus multiplying the effect of the training efforts. By doing this, we could meet the 1990 objective of the Department of Health and Human Services that "at least $95 \%$ of health care providers seeing suspected cases should be capable of diagnosing and treating all currently recognised STD" ${ }^{35}$

Finally, vaccination to provide active immunity against STDs represents the ideal control strategy. Hepatitis B vaccine is already available for high risk groups. Current research in the USA includes experimental vaccines for gonorrhoea, genital herpes, group B streptococcal infection, and cytomegalovirus infection. ${ }^{34}$ Field trials of gonorrhoea vaccine have been conducted, but preliminary reports are disappointing. Integration of effective vaccines into existing control programmes will be a major challenge for future managers if such vaccines are developed.

\section{Conclusion}

What can we say about the outlook for the prevention and control of STD in the USA? The increased attention shown to STD by patients and health care providers alike is stimulating. The expanded spectrum of organisms and syndromes makes the field especially ripe for innovative approaches. The emphasis on the consequences of STD to reproductive health has shown how these infections have far reaching effects on innocent victims. Progress towards effective control of STD will not occur overnight. The population at risk may continue to rise, the availability of new vaccines is still far away, and public health resources are not expanding as fast as STD. Yet the opportunity to make further advances has never been greater.

\section{References}

1. Anderson OW. Syphilis and society-problems of control in the United States, 1912-1964. Chicago: University of Chicago Press, $1965 ; 10$.

2. Henderson RH: Control of sexually transmitted diseases in the United States-a federal perspective. Br J Vener Dis 1977; 53: 211-5.

3. Brown ST, Wiesner PJ. Problems and approaches to the control and surveillance of sexually transmitted agents associated with pelvic inflammatory disease in the United States. Am J Obstet Gynecol 1980; 138: 1096-100.

4. Curran JW. Economic consequences of pelvic inflammatory disease in the United States. Am J Obstet Gynecol 1980; 138: 848-51.

5. World Health Organisation. Nongonococcal urethritis and other selected sexually transmitted diseases of public health importance. Tech Rep Ser 1981; No 660:142pp.

6. World Health Organisation and Pan American Health Organisation. Control of Sexually Transmitted Diseases. Report of a scientific group meeting, Washington DC, April 26-30, 1982 (in press).

7. Alderman JD. Poll on genital herpes. Washington Post/ABC News 1982 September (mimeographed).

8. Centers for Disease Control. Prevention of acquired immune deficiency syndrome (AIDS): report of inter-agency recommendations. $M M W R$ 1983;32:101-3.

9. Knox SR, Mandel B, Lazarowicz R. Profile of callers to the VD national hotline. Sex Transm Dis 1981;8:245-54.

10. Hart G. Screening to control infectious diseases: evaluation of control programs. Rev Infect Dis 1980;2:701-11.

11. Felman YM. Repeal of mandated premarital tests for syphilis: a survey of state health officers. Am J Public Health 1981; 71: $155-9$.

12. Kingon RJ, Wiesner PJ. Premarital syphilis screening: weighing the benefits. Am J Public Health 1981;71:160-3.

13. St John RK, Curran JW. Epidemiology of gonorrhea. Sex Transm Dis 1978;6:81-5. 
14. Felman YM, Snyder RR, Giordana R, Griffin J. Gonorrhea screening. Experience of a large municipal program. $N$ Y State J Med 1978; 78: 1267-75.

15. Sidhu S, Barnes R. Penicillinase-producing Neisseria gonorrhoeae-Los Angeles. MMWR 1983;32:181.

16. Jaffe $\mathrm{HW}$, Biddle $\mathrm{JW}$, Thornsberry $\mathrm{C}$, et al. National gonorrhea therapy monitoring study. In vitro antibiotic susceptibility and its correlation with treatment results. $N$ Engl J Med 1976;294: 5-9.

17. Washington AE, Mandell GL, Wiesner PJ. Treatment of sexually transmitted diseases: new additions to an old tradition. Rev Infect Dis 1982; 4 suppl 2:S727-8.

18. Brown S, Warnnissorn T, Biddle J, Panikabutra K, Traisupa A. Antimicrobial resistance of Neisseria gonorrhoeae in Bangkok: is single-drug treatment passé? Lancet 1982; ii: 1366-9.

19. Seth AD, Johnston NA. Penicillin-resistant gonococci. Lancet 1980; ii:531-3.

20. Centers for Disease Control. Current trends: gonorrhea-CDC recommended treatment schedules, 1978. MMWR 1979;28: 13-5.

21. Fletcher JD, Stratton JD, Chandler CS, et al: Penicillinresistant gonorrhea-North Carolina. MMWR 1983;32:273-4.

22. Jaffe $\mathbf{H W}$, Biddle JW, Johnson SP, Wiesner PJ. Infections due to penicillinase-producing Neisseria gonorrhoeae in the United States: 1976-1980. J Infect Dis 1981;144:191-7.

23. Centers for Disease Control. Sexually transmitted diseases: treatment guidelines, 1982. MMWR 1982;31 suppl 2:730-46.

24. Greenwood JR, Prendergast T, Ehling LR, et al. ChancroidCalifornia. MMWR 1982;31:173-5.

25. Potterat JJ, Rothenberg RB. The case finding effectiveness of a self-referral system for gonorrhea: a preliminary report. $\mathrm{Am}$ J Public Health 1977;67:174-81.

26. Handsfield HH, Sandström EG, Knapp JS, et al. Epidemiology of penicillinase-producing Neisseria gonorrhoeae infections: analysis by auxotyping and serotyping. N Engl J Med 1982;306:950-4.

27. Potterat JJ, King RD. A new approach to gonorrhea control: the asymptomatic male and incidence reduction. JAMA 1981; 245: $578-80$
28. Parra WC, Wiesner PJ, Drotman DP. Patient counseling. In: Holmes KK, et al, eds. Sexually transmitted diseases. New York; McGraw Hill (in press).

29. Graves JL, Hudgins AA, DeLung J, et al: Computerized patient flow analysis of local family planning clinics. Fam Plann Perspect 1981; 13: 164-70.

30. Centers for Disease Control. Sexually transmitted diseases summary, 1982. Atlanta: Centers for Disease Control, 1982: 1 p.

31. Centers for Disease Control. Ouality assurance guidelines for STD clinics, 1982. Atlanta: Centers for Disease Control, 1982: 66pp.

32. Stamm WE, Kaetz S, Holmes KK. Clinical training in venereology in the United States and Canada. JAMA 1982; 248:2020-4

33. Margolis $\mathrm{S}$. Initiation of the sexually transmitted diseases prevvention/training clinic program. Sex Transm Dis 1981;8:87-93.

34. National Institute of Allergy and Infectious Disease Study Group. Sexually transmitted diseases: 1980 status report. Washington DC: US Government Printing Office, 1981. (NIH Publication No 81-2213): 264pp.

35. Department of Health and Human Services. Promoting health/preventing disease: objectives for the nation. Washington, DC: US Government Printing Office, 1980; 25-9.

36. Hatcher RA, Stewart GK, Stewart F, et al. Contraceptive technology, 1982-1983. 11th ed. New York: Irvington Publishers, 1982;9-13.

37. Wiesner PJ. Developing a national strategy for the control of chlamydial infections-USA. In: Mårdh, P-A, et al, eds. Chlamydia infections. Amsterdam: Elsevier Biomedical Press, $1982 ; 437-40$.

38. Fichtner RR, Blount JH, Spencer JN. A statewide management information system for the control of sexually transmitted diseases. In: Proceedings of the seventh annual symposium on computer applications in medical care. New York: Institute of Electrical and Electronics Engineers (in press). 